\title{
ASOCIACIÓN ENTRE CONOCIMIENTOS DE ANTICONCEPCIÓN Y EMBARAZO NO PLANEADO. ESTUDIO DE CORTE TRANSVERSAL
}

\section{Association between knowledge of contraception and unplanned pregnancy. A cross-sectional study}

Sergio Augusto Cuervo-Vergara, $M D^{1}$; Jesús David Garrido-Gutiérrez, $M D^{2}$; Gladys Adriana Vélez-Álvarez, $\mathrm{MD}^{3}$; John Jairo Zuleta-Tobón, $\mathrm{MD}, \mathrm{MSc}^{4}$

Recibido: octubre 30/14 - Aceptado: agosto 27/15

\section{RESUMEN}

Objetivo: medir la asociación entre el nivel de conocimientos sobre la resolución de problemas en la utilización de los métodos anticonceptivos y la presencia de embarazo no planeado en mujeres que usan métodos anticonceptivos de corta acción. Establecer la prevalencia del embarazo no planeado y describir actitudes, percepciones y características de la atención en las actividades de planificación familiar.

Materiales y métodos: estudio analítico de corte transversal. Se incluyeron mujeres entre 14 y 49 años de edad, que acudieron a realizarse una prueba de embarazo a una institución de salud del sector público, de bajo nivel de complejidad, que atiende población del régimen de aseguramiento subsidiado por el Estado en la ciudad Medellín (Colombia). Antes de obtener el resultado de la prueba de embarazo se les realizó una encuesta estructurada y un test

1 Especialista en Obstetricia y Ginecología, Medellín (Colombia). sarave48@hotmail.com

Especialista en Obstetricia y Ginecología, Medellín (Colombia).

3 Especialista en Obstetricia y Ginecología; magíster en Salud Pública, Universidad de Antioquia. NACER, Departamento de Obstetricia y Ginecología, Profesor asistente, Medellín (Colombia).

4 Especialista en Obstetricia y Ginecología; magíster en Epidemiología, Universidad de Antioquia, Medellín (Colombia). NACER, Departamento de Obstetricia y Ginecología, Profesor titular. jjzuleta@une.net.co acerca de su conocimiento para resolver situaciones que pueden afectar la efectividad de los métodos de anticoncepción dependientes de la mujer.

Resultados: se realizaron 471 encuestas, el 75,2\% de las mujeres no planeaban embarazarse y el $57 \%$ tuvieron un embarazo no planeado. La mediana del nivel de conocimientos fue del 50\% (p25: 37,5\%, p75: 62,5\%). La razón de prevalencias de embarazo no planeado con nivel medio y alto de conocimientos fue 0,56 (IC 95\%: 0,34-0,92).

Conclusión: el conocimiento sobre la resolución de los problemas que afectan la efectividad de los métodos anticonceptivos que dependen del buen uso de la mujer para garantizar esa efectividad se asocia a menor frecuencia de embarazo no planeado. Palabras clave: embarazo no planeado, planificación familiar.

\section{ABSTRACT}

Objective: To measure the association between the degree of knowledge on how to solve problems associated with the use of contraceptive methods and the presence of unplanned pregnancies in women using short-acting contraception; to determine the prevalence of unplanned pregnancies; and to describe attitudes, perceptions and characteristics in relation to family planning activities. 
Materials and methods: Cross-sectional analytical study conducted in women between 14 and 49 years of age coming for a pregnancy test to a public, low-complexity healthcare institution in Medellín, Colombia, that provides care to a population covered under a state-subsidized healthcare system. Before delivering the result of the pregnancy test they were given a structured survey and a test to measure their knowledge for solving situations that might affect the effectiveness of contraception methods under the woman's control.

Results: Of the 471 women surveyed, $75.2 \%$ were not planning to become pregnant and $57 \%$ had an unplanned pregnancy. The median knowledge level was 50\% (p25: 37.5\%, p75: 62.5\%). The prevalence ratio of unplanned pregnancy with an intermediate or high level of knowledge was 0.56 (95\% CI 0.34-0.92).

Conclusion: Knowledge about how to solve problems regarding contraceptive methods that depend for their effectiveness on the appropriate use by the woman is associated with a lower frequency of unplanned pregnancies.

Key words: Unplanned pregnancy; family planning.

\section{INTRODUCCIÓN}

El embarazo no planeado es un problema de salud pública por las repercusiones sociales y de salud sobre las madres y sus niños. En América Latina, del 17 al $35 \%$ de estos embarazos terminan en abortos inseguros (1). Las mujeres con embarazo no planeado utilizan menos los servicios de salud materno-neonatales y acuden menos a los controles prenatales (2); consumen más tabaco y licor (3) y menos vitaminas durante el embarazo (4); lactan con menor frecuencia y durante una menor cantidad de tiempo que las mujeres que tienen embarazos planeados (5). Los niños nacidos de mujeres con embarazos no planeados tienen un mayor riesgo de tener bajo peso al nacer, de ser pequeños para la edad gestacional, de ser prematuros (6), de padecer depresión, de ser víctimas de maltrato infantil y de tener menor autoestima y mayor compromiso cognoscitivo y del bienestar psicológico (2).

En Colombia, la tasa global de fecundidad de 2,1 es superior a la deseada de 1,6 hijos por mujer, y la prevalencia de embarazo no planeado está entre 52 y $67 \%(7,8)$. En la literatura se encuentran diferentes factores asociados al embarazo no planeado; sin embargo, los hallazgos no son consistentes entre las investigaciones: edad materna, número de partos previos, bajo nivel socioeconómico, residencia urbana, bajo nivel educativo y socioeconómico, y falta de unión de pareja (9). Independientemente de estos factores, se sabe que una proporción importante de los embarazos no planeados ocurre en mujeres que están utilizando métodos anticonceptivos, pero los usan de manera incorrecta o de manera no consistente $(10,11)$, por tanto, la prevención primaria del embarazo no planeado debe incluir un plan para prevenir fallas del método y educación sobre el empleo adecuado de los anticonceptivos por parte de las usuarias (12).

Algunos estudios han evaluado el conocimiento de las usuarias sobre aspectos como existencia, mecanismos de acción o efectividad de los diferentes métodos (13); sin embargo, se tiene poca información con respecto al conocimiento específico de las mujeres para la solución de problemas que se presentan durante el uso de los anticonceptivos y que pueden afectar su efectividad $(11,14)$. Incluso en países desarrollados, se ha encontrado que las mujeres que solicitan terminación electiva del embarazo y que refirieron que estaban utilizando métodos anticonceptivos al momento de la fecundación, reportan como explicaciones a las fallas la ruptura del preservativo, el olvido de píldoras o la presencia de diarrea durante su uso, el desplazamiento del diafragma o la expulsión del dispositivo intrauterino (15), situaciones sobre las cuales las mujeres deberían tener conocimientos para resolverlas y prevenir un embarazo.

El objetivo principal de esta investigación fue evaluar la asociación entre la incidencia del embarazo no planeado y el nivel de conocimientos sobre 
la utilización correcta y resolución de problemas que afectan la efectividad de los métodos anticonceptivos de corta acción. Objetivos secundarios fueron estimar la prevalencia de embarazo no planeado y describir actitudes y percepciones con respecto a la atención en las actividades de planificación familiar y características clínicas y de la atención de las mujeres que sospechan que están en embarazo.

\section{MATERIALES Y MÉTODOS}

Se realizó un estudio observacional analítico de corte transversal, durante diez meses en el año 2012, en la Unidad Hospitalaria Hermenegildo de Fex, del municipio de Medellín, entidad de salud del sector público, de bajo nivel de complejidad, que asume la atención obstétrica de la mayor parte de la población del régimen subsidiado y no vinculada de la ciudad. Se invitó a participar a todas las mujeres entre los 14 y 49 años de edad que se realizaron una prueba de embarazo durante el periodo de estudio. Se excluyeron las mujeres con trastorno psiquiátrico o mental que les impidiera dar respuesta a los cuestionarios y las que estaban en seguimiento por antecedente de embarazo ectópico o enfermedad molar.

Procedimiento. Una encuestadora entrenada y no perteneciente al equipo investigador explicó de manera individual a las potenciales participantes el objetivo del estudio y los procedimientos, e hizo énfasis en la confidencialidad de los datos. Todas las mujeres que aceptaron participar firmaron un consentimiento informado y, antes de conocer el resultado de la prueba de embarazo, respondieron una encuesta estructurada y un cuestionario de conocimientos sobre la solución a diferentes situaciones que modifican la efectividad de los métodos anticonceptivos. Se aplicó un cuestionario con ocho preguntas abiertas para evaluar el conocimiento sobre la fecha de inicio y continuidad de los anticonceptivos inyectables, la resolución del olvido de uso de anticonceptivos orales, la conducta ante la ruptura de un preservativo durante la relación, el uso de la anticoncepción de emergencia y la utilización de prácticas tradicionales como el lavado vaginal poscoito para resolver alguna potencial falla del método. Esta prueba no está validada, pero da respuesta a los motivos de falla reconocidos en la literatura (14). Las primeras diez encuestas se consideraron prueba piloto y no fue necesario modificar ningún elemento de la encuesta o del cuestionario, los datos recolectados por la encuestadora se ingresaron en una hoja de Excel que posteriormente fue verificada por dos de los autores.

La variable de exposición fue el conocimiento para resolver las situaciones dependientes de la mujer que modifican la efectividad de los métodos de planificación de corta acción. Este nivel de conocimiento sobre métodos de planificación se calificó sobre $100 \%$ y se categorizó de manera arbitraria como bajo (menor de 40\%), medio (entre 40 y $80 \%$ ) y alto (mayor de $80 \%$ ), a partir de la calificación obtenida en el cuestionario. Como posibles factores adicionales asociados al embarazo no planeado se evaluaron la postura religiosa; la relación de pareja, en la cual se consideró como estable la convivencia de pareja igual o mayor de dos años; la escolaridad, considerada baja para estudios hasta secundaria incompleta; los ingresos mensuales; la condición socioeconómica según el nivel del SISBEN, clasificación oficial que existe en Colombia mediante la cual los hogares se califican según las condiciones de la vivienda y los ingresos económicos del grupo familiar, de nivel 1 a nivel 6, donde el 1 es el menor y 6 el máximo; las personas encargadas de la crianza; el antecedente de violencia intrafamiliar o sexual; el uso del tiempo libre, asumido adecuado como la realización de actividades deportivas o culturales; la presencia de proyecto de vida, entendida como el reconocimiento de tener objetivos plausibles por desarrollar en los siguientes años; el tabaquismo, como el consumo de al menos cinco cigarrillos a la semana; el consumo de alcohol, como la ingesta de al menos dos tragos al menos dos veces por semana; el consumo de sustancias ilegales psicoactivas, y la dificultad para adquirir los métodos de planificación. Se evaluó la percepción general que las usuarias tienen sobre la calidad del funcionamiento de las 
instituciones donde reciben atención médica. El desenlace del estudio fue el embarazo no planeado y en él se incluyó a toda mujer que tuvo prueba de embarazo positiva y que había respondido en la encuesta que no estaba buscando gestación en ese momento ni la había buscado en los seis meses previos, independientemente de que estuviera o no utilizando métodos anticonceptivos (16).

La prevalencia de embarazo no planeado se obtuvo al dividir el número de mujeres que en el seguimiento tuvieron prueba de embarazo positiva y que no estaban buscando gestación, por el número total de mujeres que realizó la encuesta, previo a la realización de la prueba.

La asociación entre el nivel de conocimiento y el embarazo no planeado se evaluó en la población susceptible de que los conocimientos evaluados en el cuestionario afectaran la aparición de embarazo, es decir, en la que no planea un embarazo y utiliza métodos de corta acción (anticonceptivos orales combinados, inyectables mensuales, de barrera y naturales).

Los supuestos para el cálculo del tamaño de la muestra fueron: frecuencia esperada de conocimiento alto en el grupo de mujeres con embarazo planeado de un $20 \%$ y en el grupo con embarazo no planeado de $10 \%$, relación de 1 mujer con embarazo planeado por cada 2 mujeres con embarazo no planeado, poder del $80 \%$ y nivel de significación del 5\%; al aplicar la fórmula con corrección de continuidad se requerían 474 mujeres (302 con embarazo no planeado y 172 con embarazo planeado). Se realizó muestreo consecutivo.

Para la comparación de las variables cualitativas según exposición (nivel de conocimiento medio/alto y nivel bajo) se utilizó la prueba de chi cuadrado, o la exacta de Fischer cuando alguna casilla tenía valores esperados menores a 5 . La variable continua que tuvo distribución normal se presenta con media y desviación estándar, y se compara entre los grupos con la prueba t de Student, y las variables cuantitativas que no tuvieron tal distribución y las discretas, se presentan con mediana y percentiles
25 y 75, y se comparan con la prueba U de Mann Whitney. Para evaluar la asociación del nivel de conocimientos en tres categorías (bajo, medio y alto) con la presencia de embarazo, se aplicó una prueba chi cuadrado de tendencia. Para evaluar la asociación entre los niveles de conocimiento (alto/ medio frente a bajo) en la aparición de embarazo, se calculó la razón de prevalencias (RP) cruda con el respectivo intervalo de confianza del $95 \%$. A fin de ajustar el potencial efecto de las otras variables preestablecidas en el protocolo, se implementó un modelo de regresión logística con el método enter, previo descarte de la presencia de multicolinealidad entre ellas. En el protocolo se había definido que se incluirían la edad y las otras características que tuvieran un valor $\mathrm{p}<0,25$ en el análisis univariado, respetando la relación del número de variables por número de desenlaces, según lo recomendado en la literatura. Los datos se ingresaron en un formulario en el programa Microsoft Excel 2007 y los análisis se realizaron con el programa estadístico SPSS 20 y el programa para análisis epidemiológico Epidat 3.1.

Se contó con la aprobación del Comité de Ética de la Empresa Social del Estado Metrosalud para implementar la investigación. Se garantizó la confidencialidad de la información, y el resultado de la prueba de embarazo se ligó a las encuestas exclusivamente con el número de identificación. Para las pacientes menores de edad, debido al tema de investigación, no se requirió consentimiento de los padres o representantes. La jurisprudencia colombiana ha aceptado que en temas de anticoncepción y sexualidad se respete la autonomía del menor.

\section{RESULTADOS}

Se realizaron 471 encuestas, dos mujeres no informaron sobre la planeación del embarazo y de otra no se anotó el resultado de la prueba de embarazo. El $75,3 \%$ no planeaban embarazarse en ese momento (353 de 469 que dieron esta respuesta) y el 60,3\% (284) estaban usando algún método de planificación familiar; 158 mujeres tuvieron prueba de embarazo positiva (158/470; 33,6\%), y de ellas, 90 no lo 
habían planeado, lo que representa una prevalencia de embarazo no planeado del $57 \%$.

De las 353 mujeres que reconocieron que no estaban planeando un embarazo, 252 (71,5\%) estaban planificando en el momento de la encuesta y 90 de ellas $(25,5 \%$ del total, $35,7 \%$ de las que estaban planificando) tuvieron prueba de embarazo positiva. Treinta de las mujeres que expresaron que estaban planeando un embarazo declararon que estaban planificando en ese momento (25,9\%). De manera similar, 101 de las mujeres que no estaban planeando el embarazo no estaban planificando $(28,6 \%)$. En la figura 1 se discrimina la población estudiada según la planeación del embarazo, el uso de anticoncepción y el resultado de la prueba. Los métodos con más fallas fueron los de barrera y los anticonceptivos orales combinados (tabla 1).

El $93 \%$ reportó que no tenía dificultades para la adquisición de los métodos anticonceptivos. Los motivos de dificultad de las 33 que reportaron fueron: sentimientos de pena y vergüenza $(39,4 \%)$, costos altos (21,2\%), desconocimiento (15,2\%), indecisión (9,1\%), falta de disponibilidad (9,1\%), otros motivos (3\%) y $3 \%$ no explicó. La percepción acerca del funcionamiento de los servicios de salud por parte de las mujeres que estaban planificando al momento de la encuesta es buena para un $57 \%$ (162), regular para un 23,6\% (67) y mala un 4,6\% (13).

De las 187 mujeres que no estaban planificando, 138 (73.8\%) lo habían hecho previamente, 69 (36,9\%) referían haber asistido a asesorías sobre el tema, $75(40,1 \%)$ no asistían a asesorías a pesar de conocer de su existencia y 43 (23.0\%) expresaron no conocer la existencia de las asesorías sobre los métodos de planificación. Entre estas mujeres, los principales obstáculos para la adquisición de los métodos son los sentimientos de pena o vergüenza $(43,7 \%)$ y los altos costos (25\%).

Nivel de conocimientos y embarazo no planeado. La mediana del nivel de conocimientos fue del $50 \%$ (p25: 37,5\%, p75: 62,5\%), sin mayores diferencias entre las no usuarias $(46,2 \%)$, y las usuarias de métodos de corta $(48,9 \%)$ o larga acción $(47,4 \%)$ $(\mathrm{p}=0,956)$.

De las 252 mujeres que no planeaban embarazo y estaban planificando, 188 lo estaban haciendo con métodos de corta acción, y de estas últimas, 45 tuvieron prueba de embarazo positiva. Al analizar el nivel de conocimiento en las tres categorías iniciales planeadas, se confirmó una tendencia estadísticamente significativa de menor frecuencia de embarazo no planeado a mayor nivel de conocimientos

Figura 1.

\section{Relación entre la planeación del embarazo, el uso de anticoncepción y} el resultado de la prueba de embarazo

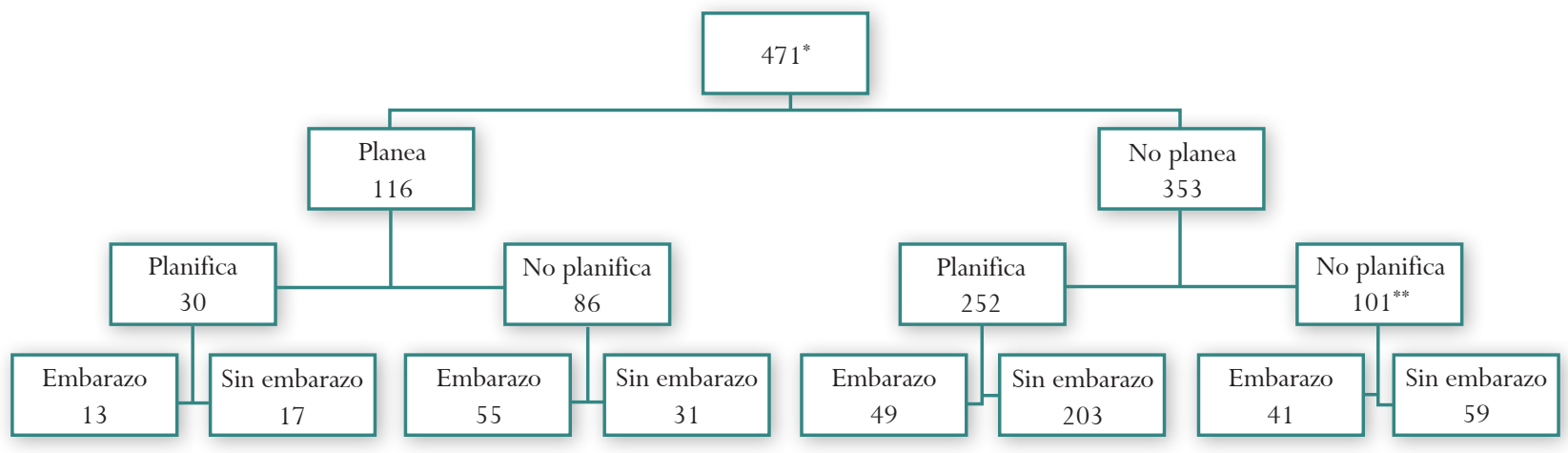

* 2 no dieron respuesta a la planeación.

** 1 paciente sin resultado de PIE. 


\begin{tabular}{|c|c|c|c|}
\hline \multicolumn{4}{|c|}{$\begin{array}{c}\text { Tabla } 1 . \\
\text { Prueba de embarazo positiva según el método empleado por las } \\
\text { embarazo y estaban planificando }\end{array}$} \\
\hline & $\begin{array}{c}\text { Prueba de } \\
\text { embarazo } \\
\text { positiva }\end{array}$ & Usuarias & $\begin{array}{l}\text { Proporción de fallas } \\
\text { (\%) }\end{array}$ \\
\hline Barrera & 19 & 50 & 38,0 \\
\hline Anticonceptivos orales combinados & 15 & 54 & 27,8 \\
\hline Inyectables & 12 & 83 & 14,5 \\
\hline Quirúrgico & 2 & 26 & 7,7 \\
\hline DIU & 1 & 18 & 5,6 \\
\hline Implantes & 0 & 20 & 0,0 \\
\hline Natural & 0 & 1 & 0,0 \\
\hline Total & 49 & 252 & 19,4 \\
\hline
\end{tabular}

$(\mathrm{p}=0,004)$ (tabla 2). Dos de estas mujeres no resolvieron la prueba de conocimientos, por tanto, no se incluyen en el análisis de factores asociados al embarazo no planeado en usuarias de métodos de corta acción.

En las 11 mujeres con nivel de conocimiento alto que utilizaban métodos de corta acción, no se presentó ningún embarazo, por esta razón, se agruparon con las de la categoría con nivel medio de conocimientos. Se presentaron 22 (20,2\%) embarazos no planeados entre las 109 mujeres con nivel medio de conocimientos, para una proporción del 18,3\% de embarazos entre las 120 mujeres de la categoría conjunta de conocimientos medio y alto y 23 (34,8\%) entre las 66 de conocimiento bajo (RP $=0,56$; IC $95 \%: 0,34-0,92)$.
Se compararon los grupos de conocimiento alto/medio frente a bajo, para evaluar si diferían en otras características que pudieran afectar la aparición del embarazo no planeado, y se encontró una asociación estadísticamente significativa con el nivel de escolaridad y el proyecto de vida (tabla 3). Igualmente, se compararon los grupos según la presencia o ausencia de embarazo no planeado (tabla 4), y se hizo ajuste de la asociación por edad, falta de proyecto de vida, violencia intrafamiliar y antecedente de violencia sexual, características planteadas en el protocolo como potenciales variables de confusión y que cumplieron con el criterio estadístico preestablecido. La asociación entre nivel de conocimiento y embarazo continuó siendo estadísticamente significativa $(\mathrm{OR}$ crudo $=0,42 ; \mathrm{IC}$

\section{Tabla 2.}

Nivel de conocimientos sobre resolución de problemas con el método entre las mujeres que utilizan métodos de corta acción y no planeaban el embarazo

\begin{tabular}{|c|c|c|}
\hline $\begin{array}{c}\text { Nivel de conocimiento } \\
(\%)\end{array}$ & $\begin{array}{c}\text { Prueba de embarazo positiva (\%) } \\
(45)\end{array}$ & $\begin{array}{c}\text { Prueba de embarazo negativa (\%) } \\
(141)\end{array}$ \\
\hline Alto & $0(0,1)$ & $11(100,0)$ \\
\hline Medio & $22(20,2)$ & $87(79,8)$ \\
\hline Bajo & $23(34,8)$ & $43(65,2)$ \\
\hline
\end{tabular}




\begin{tabular}{|c|c|c|c|}
\hline & $\begin{array}{c}\text { Alto/Medio } \\
(120)\end{array}$ & $\begin{array}{l}\text { Bajo } \\
(66)\end{array}$ & $p$ \\
\hline Prueba de embarazo positiva & $22(18,3)$ & $23(34,8)$ & 0,012 \\
\hline Edad $^{\circ}$ & $22,5(6,4)$ & $22,7(7,2)$ & 0,876 \\
\hline Gestaciones + & $1(0-2)$ & $1(0-2)$ & 0,715 \\
\hline Hijos vivos + & $1(0-1)$ & $1(0-2)$ & 0,459 \\
\hline Edad inicio de relaciones + & $16(15-17)$ & $15(14-17)$ & 0,099 \\
\hline Adolescente & $45(37,5)$ & $27(40,9)$ & 0,648 \\
\hline $\begin{array}{l}\text { Postura religiosa } \\
\text { Practicante } \\
\text { No practicante } \\
\text { No religioso }\end{array}$ & $\begin{array}{c}77(65,3) \\
38(32,2) \\
3(2,5)\end{array}$ & $\begin{array}{c}38(58,5) \\
25(38,5) \\
2(3,1)\end{array}$ & 0,661 \\
\hline Relación no estable & $69(80,0)$ & $39(56,0)$ & 0,833 \\
\hline Bajo nivel de escolaridad & $56(46,7)$ & $50(75,8)$ & $<0,0001$ \\
\hline $\begin{array}{l}\text { Ingresos mensuales } \\
\text { Menos de } 1 \text { SMLV } \\
\text { Entre } 1 \text { y } 2 \text { SMLV } \\
\text { Entre } 2 \text { y } 3 \text { SMLV } \\
\text { Más de } 3 \text { SMLV* }\end{array}$ & $\begin{array}{c}88(73,3) \\
29(24,2) \\
2(1,7) \\
1(0,8)\end{array}$ & $\begin{aligned} 52 & (80,0) \\
11 & (16,9) \\
2 & (3,1) \\
0 & (0,0)\end{aligned}$ & 0,532 \\
\hline $\begin{array}{l}\text { Nivel del Sisben } \\
0 \\
1 \\
2 \\
3\end{array}$ & $\begin{array}{c}12(10,0) \\
66(55,0) \\
37(30,8) \\
5(4,2)\end{array}$ & $\begin{array}{c}13(20,0) \\
37(55,9) \\
11(16,9) \\
4(6,2)\end{array}$ & 0,085 \\
\hline $\begin{array}{l}\text { Crianza } \\
\text { Ambos padres } \\
\text { Alguno de los padres } \\
\text { Abuelos } \\
\text { Otros }\end{array}$ & $\begin{array}{c}58(48,3) \\
46(38,3) \\
9(7,5) \\
7(5,8)\end{array}$ & $\begin{array}{c}33(50,0) \\
29(40,3) \\
3(4,5) \\
1(1,5)\end{array}$ & 0,099 \\
\hline Sin apoyo familiar & $8(6,7)$ & $6(9,1)$ & 0,549 \\
\hline Con antecedente de violencia familiar & $4(3,3)$ & $5(7,5)$ & $0,287^{*}$ \\
\hline Antecedente de violencia sexual & $5(4,2)$ & $5(7,6)$ & $0,330^{*}$ \\
\hline Sin adecuado uso del tiempo libre & $49(40,8)$ & $22(33,3)$ & 0,314 \\
\hline Sin proyecto de vida claro & $31(25,8)$ & $29(43,9)$ & 0,011 \\
\hline Tabaquismo & $10(8,0)$ & $6(9,1)$ & 0,860 \\
\hline Consumo de alcohol & $20(16,7)$ & $6(9,1)$ & 0,154 \\
\hline Consumo de sustancias psicoactivas & $2(1,7)$ & $1(1,5)$ & $1,000^{*}$ \\
\hline Relaciones sexuales esporádicas & $14(12,1)$ & $4(6,2)$ & 0,202 \\
\hline Dificultad para la adquisición de los métodos de anticoncepción & $7(5,8)$ & $4(6,1)$ & 1,000 \\
\hline
\end{tabular}

+Valores expresados en percentil 50 (Percentil 25 - Percentil 75).

${ }^{\circ}$ Valores expresados en media (desviación estándar).

* Prueba exacta de Fischer. 
95\%: 0,21-0,83), con mínima variación con el ajuste $(\mathrm{OR}$ ajustado $=0,47$; IC 95\%: 0,23-0,96) (tabla 4).

\section{DISCUSIÓN}

En esta investigación se encontró que más de la mitad de los embarazos son no planeados, con la diferencia con respecto a otras investigaciones de que en este estudio se indagó sobre esa planeación antes de que las mujeres conocieran el resultado de la prueba de embarazo. La expectativa de las mujeres que saben que están en embarazo o que ya han tenido su parto puede sesgar la respuesta con respecto a la real planeación y aceptación del mismo $(10,11)$. A pesar de la diferencia en la elección del denominador para realizar el cálculo, esta prevalencia es similar al $57 \%$ estimado para Colombia en la Encuesta Nacional de Demografía y Salud (ENDS), en la cual se hace la estimación a partir de los embarazos ya confirmados (7). La frecuencia de este problema en el mundo fluctúa entre 38\% en Europa y $86 \%$ en África; para Latinoamérica se calcula que es del $58 \%$, con amplias variaciones entre los países (17).

Nuestro estudio encontró que existe una asociación entre el mayor nivel de conocimientos acerca de la resolución de situaciones específicas que afectan la efectividad del método y una menor frecuencia de embarazos no planeados. Estudios previos han evaluado el conocimiento sobre la existencia de los diferentes métodos o sobre aspectos específicos de los mismos, como los mecanismos de acción, las diferencias en efectividad y la posibilidad de inducir efectos secundarios o efectos benéficos, entre otros $(13,18)$. Se reconoce que el conocimiento de estos aspectos es muy importante al momento de tomar la decisión del inicio de un método, pero no tiene influencia sobre la efectividad del mismo. La asociación encontrada no se modificó de manera importante al tener en cuenta otros factores que pudieran influir; sin embargo, el modelo muestra que las características evaluadas explican poca de la variabilidad del resultado (tabla 4), por tanto, se puede afirmar que existen otras características no evaluadas en este estudio, las cuales podrían explicar la ocurrencia del embarazo no planeado en esta población.

En general, se acepta que la ocurrencia de los embarazos no planeados se da principalmente por la falta de uso, el uso incorrecto o la falla de los métodos anticonceptivos (11). En este estudio, una alta proporción de mujeres reconoció que no tenía dificultades para adquirir los diferentes métodos de planificación, que tenían acceso a programas de asesoría e incluso que previamente habían utilizado alguno, lo cual sugiere que la falta de disponibilidad del método no es una causa importante del problema del embarazo no planeado en esta población. Este hallazgo es consistente con un conocimiento universal de la existencia y el uso, al menos una vez, de algún método en el $85 \%$ de las mujeres colombianas, informado en la ENDS (19).

A pesar del carácter transversal de esta investigación, al momento de evaluar los factores no se conocía el desenlace, y no hay duda de que los factores contemplados lo precedían, por tanto, con las debidas precauciones, se pueden al menos explorar asociaciones etiológicas (20). No se encontró asociación entre embarazo no planeado y las condiciones de vida analizadas, lo que se puede explicar por las particularidades de este fenómeno. Algunos autores proponen que la intención de impedir un embarazo no planeado es más compleja que la intención de proceder a un embarazo planeado (21). El paso de no intención a intención, de planear a no planear, no es una cuestión de todo o nada, es un continuo que pasa de ser totalmente involuntario a no planeado, de ahí a voluntario y por último a totalmente planeado (22). Específicamente, aun cuando la intención inequívoca de una mujer sea impedir un embarazo, su actitud hacia la anticoncepción tiene que ser totalmente segura si ella no quiere concebir (22).

Dos terceras partes de los embarazos de mujeres que no lo estaban planeando se presentaron en adultas, el 86,7\% de los embarazos de usuarias de anticoncepción ocurrieron en las que estaban empleando métodos que dependen del adecuado 


\begin{tabular}{|c|c|c|c|c|}
\hline & $\begin{array}{c}\text { Embarazo } \\
(45) \\
\end{array}$ & $\begin{array}{l}\text { Sin embarazo } \\
(141)\end{array}$ & $P$ & $\begin{array}{l}\text { OR ajustado } \\
\text { (IC 95\%) }\end{array}$ \\
\hline $\begin{array}{l}\text { Nivel de conocimientos } \\
\text { Alto/medio } \\
\text { Bajo }\end{array}$ & $\begin{array}{l}22(49,8) \\
23(51,1)\end{array}$ & $\begin{array}{l}98(69,5) \\
43(30,5)\end{array}$ & 0,012 & $0,47(0,23-0,96)$ \\
\hline Edad $^{\circ}$ & $24,0(7,1)$ & $22,1(6,5)$ & 0,101 & $1,03(0,98-1,08)$ \\
\hline Gestaciones + & $1(0-2)$ & $1(0-2)$ & 0,715 & \\
\hline Hijos vivos + & $1(0-2)$ & $1(0-1)$ & 0,885 & \\
\hline Edad inicio de relaciones + & $16(15-17)$ & $15(14-17)$ & 0,364 & \\
\hline Adolescente & $15(33,3)$ & $57(40,4)$ & 0,395 & \\
\hline $\begin{array}{l}\text { Postura religiosa } \\
\text { Practicante } \\
\text { No practicante } \\
\text { No religioso }\end{array}$ & $\begin{aligned} 27 & (61,4) \\
15 & (34,1) \\
2 & (4,5)\end{aligned}$ & $\begin{array}{c}88(63,3) \\
48(34,5) \\
3(2,2)\end{array}$ & 0,698 & \\
\hline Relación no estable & $21(46,7)$ & $57(40,4)$ & 0,460 & \\
\hline Bajo nivel de escolaridad & $26(57,8)$ & $80(56,7)$ & 0,902 & \\
\hline $\begin{array}{l}\text { Ingresos mensuales } \\
\text { Menos de } 1 \text { SMLV } \\
\text { Entre } 1 \text { y } 2 \text { SMLV } \\
\text { Entre } 2 \text { y } 3 \text { SMLV } \\
\text { Más de } 3 \text { SMLV }\end{array}$ & $\begin{array}{l}33(75,0) \\
9(20,5) \\
2(4,5) \\
0(0,0)\end{array}$ & $\begin{array}{c}107(75,9) \\
31(22,0) \\
2(1,4) \\
1(0,7)\end{array}$ & 0,600 & \\
\hline $\begin{array}{l}\text { Nivel del SISBEN } \\
0 \\
1 \\
2 \\
3\end{array}$ & $\begin{array}{c}5(11,4) \\
23(52,3) \\
14(31,8) \\
2(4,5)\end{array}$ & $\begin{array}{c}20(14,2) \\
80(56,7) \\
34(24,1) \\
7(5,0)\end{array}$ & 0,657 & \\
\hline $\begin{array}{l}\text { Crianza } \\
\text { Ambos padres } \\
\text { Algunos de los padres } \\
\text { Abuelos } \\
\text { Otros }\end{array}$ & $\begin{array}{c}23(51,1) \\
18(40,0) \\
2(4,4) \\
2(4,4)\end{array}$ & $\begin{array}{c}68(48,2) \\
57(40,4) \\
10(7,1) \\
6(4,3)\end{array}$ & 0,933 & \\
\hline Sin apoyo familiar & $2(4,4)$ & $12(8,5)$ & 0,368 & \\
\hline Con antecedente de violencia familiar & $4(8,9)$ & $5(3,5)$ & 0,146 & $1,6(0,31-7,9)$ \\
\hline Antecedente de violencia sexual & $5(11,1)$ & $5(3,5)$ & 0,05 & $2,6(0,57-11,5)$ \\
\hline Sin adecuado uso del tiempo libre & $15(33,3)$ & $56(39,7)$ & 0,443 & \\
\hline Sin proyecto de vida claro & $19(42,2)$ & $41(29,1)$ & 0,101 & $1,54(0,74-3,24)$ \\
\hline Tabaquismo & $4(8,9)$ & $12(8,5)$ & 0,937 & \\
\hline Consumo de alcohol & $7(15,6)$ & $19(13,5)$ & 0,726 & \\
\hline Consumo de sustancias psicoactivas & $2(4,4)$ & $1(0,7)$ & 0,083 & \\
\hline Relaciones sexuales esporádicas & $5(11,1)$ & $13(9,6)$ & 0,763 & \\
\hline $\begin{array}{l}\text { Dificultad para la adquisición de los } \\
\text { métodos de anticoncepción }\end{array}$ & $1(2,2)$ & $10(7,1)$ & 0,228 & \\
\hline $\begin{array}{l}\text { Satisfacción con el método } \\
\text { Satisfecha } \\
\text { Indiferente } \\
\text { Insatisfecha }\end{array}$ & $\begin{aligned} 29 & (64,4) \\
4 & (8,9) \\
12 & (26,7)\end{aligned}$ & $\begin{array}{l}99(70,2) \\
17(12,1) \\
25(17,7)\end{array}$ & 0,399 & \\
\hline
\end{tabular}

* Prueba de Hosmer-Lemeshow: $\mathrm{p}=$ 0,062; R cuadrado de Nagelkerke = 0,095. 
uso por parte de la usuaria para garantizar su eficacia, y hubo un conocimiento bajo sobre ese uso adecuado. Jaccard, citado por Black et al., propone que, a menudo, la mujer evalúa y reevalúa los riesgos y beneficios del embarazo y de la anticoncepción (11). Por otro lado, los métodos de larga acción son mucho más efectivos, incluso en situaciones donde se está realizando acompañamiento a las mujeres que utilizan métodos de corta acción (23). Nuestros resultados, aunados a ese conocimiento, permiten proponer que se haga mayor énfasis en métodos de larga acción que obvian esa dependencia de la mujer para lograr efectividad. Por otro lado, el hecho de que más de la mitad de los embarazos no planeados se presentaron en mujeres adultas (tabla 4), invita a que se involucre más a este grupo poblacional en las diferentes estrategias y campañas de educación en planificación familiar, generalmente dedicadas a la adolescencia. Por último, el reconocimiento por parte de algunas mujeres de los sentimientos de pena o vergüenza para acceder a los métodos de planificación, y de que otras no asisten a pesar de reconocer la presencia de los servicios, debe alertar al personal de la salud a buscar alternativas de convocatoria y a buscar una adecuada relación con las mujeres, fundamental para superar estas barreras (24).

Nuestro estudio tiene como fortaleza el momento de la evaluación con respecto al conocimiento del resultado de la prueba, con lo cual se previenen sesgos en los juicios que ellas pudieran hacer si estuvieran influenciadas por el conocimiento de su real condición con respecto al embarazo. Por otro lado, presenta la debilidad de haber utilizado formularios estructurados, con lo cual se limita la oportunidad de detectar factores que influencian los comportamientos de las mujeres.

\section{CONCLUSIÓN}

Se encontró que la frecuencia de embarazo no planeado en la población estudiada es de un 57\%, y que el conocimiento sobre la resolución de los problemas que afectan la efectividad de los métodos anticonceptivos que dependen del buen uso de la mujer para garantizarla se asocia a menor frecuencia de embarazo no planeado.

\section{AGRADECIMIENTOS}

A la Empresa Social del Estado Metrosalud y en especial a la Unidad Hospitalaria Hermenegildo de Fex por haber autorizado y facilitado la implementación de este estudio.

\section{FINANCIACIÓN}

Esta investigación hizo parte de una convocatoria externa de la Empresa Social del Estado Metrosalud; tuvo financiación parcial de NACER Salud Sexual y Reproductiva, de la Universidad de Antioquia.

\section{REFERENCIAS}

1. Langer A. Unwanted pregnancy: impact on health and society in Latin America and the Caribbean. Rev Panam Salud Publica. 2002;11:192-204.

2. Logan C, Holcombe E, Manlove J, Ryan S. The Consequences of Unintended Childbearing. A white paper; 2007.

3. Walker MJ, Al-Sahab B, Islam F, Tamim H. The epidemiology of alcohol utilization during pregnancy: an analysis of the Canadian Maternity Experiences Survey (MES). BMC Pregnancy Childbirth. 2011; 11:52.

4. Bitto A, Gray RH, Simpson JL, Queenan JT, Kambic RT, Perez A, et al. Adverse outcomes of planned and unplanned pregnancies among users of natural family planning: a prospective study. Am J Public Health. 1997;87:338-43.

5. Taylor JS, Cabral HJ. Are women with an unintended pregnancy less likely to breastfeed? J Fam Pract. 2002;51:431-6.

6. Shah PS, Balkhair T, Ohlsson A, Beyene J, Scott F, Frick C. Intention to become pregnant and low birth weight and preterm birth: a systematic review. Matern Child Health J. 2011;15:205-16.

7. Profamilia. Preferencias sobre fecundidad y demanda de planificación familiar. Encuesta Nacional de 
Demografía y Salud 2010. Bogotá; 2011. p. 167-90.

8. Prada E, Sing S, Remez L, Villarreal C. Unintended Pregnancy And Induced Abortion in Colombia. Causes and consequences. Guttmacher Institute, editor. New York: Guttmacher Institute; 2011. p. 72.

9. Ikamari L, Izugbara C, Ochako R. Prevalence and determinants of unintended pregnancy among women in Nairobi, Kenya. BMC Pregnancy Childbirth. 2013;13:69.

10. Palanivelu LM, Oswal A. Contraceptive practices in women with repeat termination of pregnancies. J Obstet Gynaecol. 2007;27:832-4.

11. Black KI, Gupta S, Rassi A, Kubba A. Why do women experience untimed pregnancies? A review of contraceptive failure rates. Best Pract Res Clin Obstet Gynaecol. 2010;24:443-55.

12. Taylor D, James EA. An evidence-based guideline for unintended pregnancy prevention. J Obstet Gynecol Neonatal Nurs. 2012;40:782-93.

13. Craig AD, Dehlendorf C, Borrero S, Harper CC, Rocca CH. Exploring young adults' contraceptive knowledge and attitudes: Disparities by race/ethnicity and age. Women's Health Issues. 2014;24:e281-9.

14. Schünmann C, Glasier A. Measuring pregnancy intention and its relationship with contraceptive use among women undergoing therapeutic abortion. Contraception. 2006;73:520-4.

15. Sheeder J, Tocce K, Stevens-Simon C. Reasons for ineffective contraceptive use antedating adolescent pregnancies: part 2: a proxy for childbearing intentions. Matern Child Health J. 2009;13:306-17.
16. Finer LB, Zolna MR. Unintended pregnancy in the United States: incidence and disparities, 2006. Contraception. 2011;84:478-85.

17. Singh S, Sedgh G, Hussain R. Unintended pregnancy: worldwide levels, trends, and outcomes. Stud Fam Plann. 2010;41:241-50.

18. Frost JJ, Lindberg LD, Finer LB. Young adults' contraceptive knowledge, norms and attitudes: associations with risk of unintended pregnancy. Perspect Sex Reprod Health. 2012;44:107-16.

19. Profamilia. Planificación familiar: conocimiento y uso de métodos. Encuesta Nacional de Demografía y Salud 2010. Bogotá; 2011. p. 32.

20. Lee J, Chia KS. Estimation of prevalence rate ratios for cross sectional data: an example in occupational epidemiology. Br J Ind Med. 1993;50:861-2.

21. Lifflander A, Gaydos LMD, Hogue CJR. Circumstances of pregnancy: low income women in Georgia describe the difference between planned and unplanned pregnancies. Matern Child Health J. 2007;11:81-9.

22. Zabin LS. Ambivalent feelings about parenthood may lead to inconsistent contraceptive use-and pregnancy. Fam Plann Perspect. 1999;31:250-1.

23. Winner B, Peipert JF, Zhao Q, Buckle C, Madden T, Allsworth JE, et al. Effectiveness of long-acting reversible contraception. N Engl J Med. 2012; 366:1998-2007.

24. Isaacs JN, Creinin MD. Miscommunication between healthcare providers and patients may result in unplanned pregnancies. Contraception. 2003; 68: 373-6. 